\title{
Seguridad de las fluoroquinolonas: riesgos habitualmente olvidados para el clínico
}

\author{
Claudio González M., Ruth Rosales C., Daniela Pavez, Luz M. Fuenzalida, Andrés Soto, \\ Regina Pérez, Jorge Pérez, Rafael Araos y M. Eugenia Pinto
}

\section{Safety of fluoroquinolones: risks usually forgotten for the clinician}

Quinolones are a group of widely used antimicrobials. Although they are considered safe for patients, knowledge of the safety profile is necessary so that professionals become aware of what is necessary to monitor. At the musculoskeletal level, quinolones have the potential to damage cartilage, causing even tendon rupture in infrequent cases. Hypoglycemia / hyperglycemia has been observed at the endocrine level, thus, careful monitoring of glycemia in patients with quinolone is recommended in diabetic patients. At the cardiovascular level, arrhythmias induced by these antimicrobials are rare but severe. At the level of the nervous system, the appearance of alterations of the central nervous system and the peripheral neuropathy are emphasized. When assessing the safety of quinolones, it is important to consider potential interactions with other substances (medical products). In children it is preferred not to use fluoroquinolones because of the potential risk of cartilage damage and growth, effects that do not seem to be so dramatic in the face of new evidence. Despite optimism, the safety of the treatment of these antimicrobials should be evaluated in every pediatric patient.

Key words: Fluoroquinolonas, safety, pharmaco surveillance, pediatrics.

Palabras clave: Fluoroquinolonas, seguridad, farmacovigilancia, pediatría.

\section{Introducción}

\section{L}

as quinolonas son un grupo de antimicrobianos que ha presentado un aumento progresivo de consumo ambulatorio a través de los años ${ }^{1}$. Gracias a la farmacovigilancia se han detectado reacciones adversas graves que han obligado a retirar del mercado varias fluoroquinolonas ${ }^{2}$. Por ejemplo, grepafloxacina experimentó un retiro voluntario por el efecto de prolongación del intervalo QT, complicación que exponía a los pacientes a riesgo de arritmias ventriculares graves, como la torsión de puntas ${ }^{3}$. Otro ejemplo fue el retiro de gatifloxacina, por alteraciones en los valores de glicemia ${ }^{4}$. El reporte de las reacciones adversas a medicamento a la autoridad regulatoria es un factor clave para la detección oportuna de eventos adversos en nuestra población. Si bien el grupo de las quinolonas es considerado seguro para los pacientes, el conocimiento del perfil de seguridad es necesario para que los profesionales estén en conocimiento de lo que es necesario reportar. Los efectos adversos más frecuentes de las quinolonas son los gastrointestinales (náuseas, vómitos, diarrea), las alergias y las reacciones neurológicas (mareo, cefalea, ansiedad $)^{5}$. En los últimos años algunas

\footnotetext{
* Farmacovigilancia (FV) es la ciencia que se dedica a la detección, evaluación, comprensión y prevención de los efectos adversos de los medicamentos o cualquier otro problema de salud relacionado con ellos.
}

agencias sanitarias de países desarrollados han emitido alertas de seguridad por reacciones adversas a este grupo de medicamentos y, a pesar de que estos eventos son incluidos en los folletos de información al profesional, son desconocidos para los médicos clínicos.

El objetivo de la presente revisión es dar a conocer reacciones adversas de las fluoroquinolonas que han adquirido relevancia clínica en los últimos años, con la intención de que sean detectadas en forma oportuna en la práctica clínica. Además, revisaremos aspectos de seguridad como la influencia de las interacciones entre medicamentos y el uso de este grupo en niños.

\section{Reacciones adversas a fluoroquinolonas}

Múltiples reacciones adversas han sido descritas con el uso de fluoroquinolonas y que son consideradas graves, es decir, amenazan la vida del paciente o producen incapacidad permanente o sustancial, requieren hospitalización o prolongan el tiempo de ésta ${ }^{6}$. Revisaremos el espectro de eventos graves, pero poco frecuentes, con especial énfasis en las fluoroquinolonas de uso sistémico: ciprofloxacina, levofloxacina y moxifloxacina (Tabla 1).

\section{Sistema músculo-esquelético}

Las quinolonas tienen el potencial para dañar cartílagos en modelos animales, sobre todo en las etapas de creci-
Comité de Antimicrobianos. Sociedad Chilena de Infectología.

Declaración de conflictos de interés: Ninguno. Fuente de financiamiento: Ninguno.

Recibido: 11 de octubre de 2017

Correspondencia a: Claudio González Muñoz claudio.gonzalezm@redsalud. gov.cl 


\begin{tabular}{|c|c|c|c|}
\hline Sistema involucrado & Frecuencia* & Velocidad de aparición & Factores de riesgo \\
\hline \multicolumn{4}{|l|}{ Músculo-esquelético } \\
\hline Tendinopatía & Rara & $\begin{array}{l}1^{\text {a }} \text { mes, y hasta meses tras la } \\
\text { suspensión }\end{array}$ & $\begin{array}{l}\text { Pacientes mayores de } 60 \text { años, con uso de corticosteroides y falla renal o } \\
\text { hepática }\end{array}$ \\
\hline \multicolumn{4}{|l|}{ Endocrino } \\
\hline Hipoglicemia & Rara & A los pocos días & $\begin{array}{l}\text { Pacientes con falla renal, sepsis, uso de hipoglicemiantes orales concomitantes } \\
\text { y con edad avanzada }\end{array}$ \\
\hline Hiperglicemia & Rara & A los pocos días & $\begin{array}{l}\text { Pacientes con diabetes mellitus no insulino-dependiente y en aquellos con } \\
\text { insuficiencia renal leve a moderada }\end{array}$ \\
\hline \multicolumn{4}{|l|}{ Cardiovascular } \\
\hline Arritmias & No conocida & Inicio rápido & $\begin{array}{l}\text { Hipokalemia, hipomagnesemia, disfunción renal, disfunción hepática y la } \\
\text { presencia de otros fármacos arritmogénicos }\end{array}$ \\
\hline \multicolumn{4}{|l|}{ Nervioso central } \\
\hline Convulsiones & Rara & Inicio rápido & $\begin{array}{l}\text { Antecedentes de epilepsia, uso de otros medicamentos epileptógenos, altera- } \\
\text { ciones hidroelectrolíticas e insuficiencia renal }\end{array}$ \\
\hline \multicolumn{4}{|l|}{ Nervioso periférico } \\
\hline Neuropatía sensitiva motora & No conocida & Inicio rápido & No se conocen \\
\hline
\end{tabular}

miento rápido ${ }^{7}$. El daño articular se ha establecido a partir de modelos animales, pero no se ha demostrado de forma concluyente en humanos, ni tampoco en niños que podrían tener, en teoría, mayor incidencia de este problema por su rápido desarrollo ${ }^{8}$. Otro evento similar es la tendinopatía que puede evolucionar a la rotura de tendón, principalmente el de Aquiles ${ }^{9}$. Su frecuencia de aparición es baja ${ }^{10}$ y se ha observado principalmente en pacientes mayores de 60 años, asociado al uso de corticosteroides y falla renal y hepática ${ }^{11,12}$. Este evento ocurre generalmente en el primer mes de tratamiento, aunque puede aparecer hasta varios meses de suspendido el medicamento ${ }^{13}$. Generalmente está precedida de inflamación del tendón afectado, recomendándose la suspensión del antimicrobiano al primer signo de tendinopatía para reducir su progresión hacia la rotura del tendón. Durante la administración de quinolonas, se debe monitorizar la aparición de artritis, artralgias, tendinopatía y anormalidades de la marcha, en especial en pacientes que lo han de usar por plazo mayor a una semana.

\section{Sistema endocrino}

A través de algunos estudios se ha observado que las fluoroquinolonas, en particular la retirada gatifloxacina, producen alteraciones del metabolismo de la glucosa. Su frecuencia es baja; en un estudio retrospectivo de 17.000 pacientes hospitalizados se encontraron 101 pacientes con trastornos de la glicemia a las $72 \mathrm{~h}$ de iniciar el uso de quinolonas, siendo más frecuente la hiperglicemia que la hipoglicemia ${ }^{14}$. La posibilidad de trastornos de glicemia de rápida en instauración parecen ser más alta con levofloxacina, que con las otras fluoroquinolonas ${ }^{15,16}$. Los episodios de hiperglicemia parecen ocurrir en forma más frecuente en pacientes con diabetes mellitus no insulino-dependiente y en aquellos con insuficiencia renal leve a moderada ${ }^{17}$. Por otro lado, la hipoglicemia ocurre también en pacientes no diabéticos, aunque ocurre más frecuente en pacientes con uso de hipoglicemiantes orales concomitantes, falla renal, sepsis, y con edad avanzada $^{18}$. Los mecanismos asociados a hipoglicemia no son muy claros y simularían un efecto tipo sulfonilurea de las fluoroquinolonas sobre las células pancreáticas ${ }^{19}$, y posiblemente interacciones medicamento-medicamento con la terapia hipoglicemiante ${ }^{16}$. Mientras que la hiperglicemia parece estar relacionada en modelos animales con la reducción en la liberación de insulina o aumento de la liberación de epinefrina ${ }^{20}$. En pacientes diabéticos se recomienda el control metabólico cuidadoso cuando se usan fluoroquinolonas.

\section{Sistema cardiovascular}

En los Estados Unidos de América (E.U.A.) 300.000400.000 personas fallecen cada año de muerte súbita cardiaca, siendo gran parte de esta mortalidad relacionada con arritmias ventriculares ${ }^{21}$. Las arritmias por antimicrobianos son reacciones adversas poco frecuentes, pero consideradas graves por ser una potencial amenaza a la vida del paciente ${ }^{3}$. El principal mecanismo involucrado 
es la prolongación del intervalo Q-T, un factor de riesgo de síncope, arritmias ventriculares, taquicardia ventricular polimorfa, fibrilación ventricular y de muerte súbita. Las fluoroquinolonas prolongan el intervalo Q-T a través de un bloqueo del componente rápido de la corriente rectificadora tardía de potasio en el tejido de conducción del miocardio ${ }^{22}$. Las arritmias por grepafloxacina determinaron su retiro del mercado y pusieron en alerta a este importante grupo de antimicrobianos ${ }^{23}$. Son pocos los casos reportados de arritmias ventriculares, principalmente torsión de puntas, secundarias a las actuales quinolonas $^{23-25}$.

Importante es reducir el potencial de este evento a través de la corrección de ciertos factores de riesgo como la hipokalemia, hipomagnesemia, disfunción renal, disfunción hepática y la presencia de otros fármacos que produzcan este evento o que interaccionen a nivel de citocromo P450 (amiodarona, fluconazol, eritromicina, etc.) ${ }^{26}$.

\section{Sistema nervioso central}

Son reacciones adversas bastante frecuentes, y muy diversas, como por ejemplo: ansiedad, agitación, insomnio, euforia, pesadillas, alucinaciones, psicosis y convulsiones $^{27}$. Los pacientes geriátricos, principalmente los que tienen daño neurológico de base, son más propensos a estos eventos y deben ser vigilados de forma más estricta ${ }^{28}$. Muchas veces estas reacciones son confundidas con la demencia y no es posible relacionar su presencia con el medicamento de forma clara.

La aparición de convulsiones es un evento poco frecuente con las fluoroquinolonas y debe ser considerada en el escenario de un paciente con antecedentes de epilepsia, uso de otros medicamentos epileptógenos, alteraciones hidroelectrolíticas e insuficiencia renal ${ }^{29,30}$. Su mecanismo de acción es aún desconocido, aunque parece haber una relación con un incremento del ingreso del antimicrobiano al sistema nervioso central ${ }^{29,31}$.

\section{Sistema nervioso periférico}

A partir de los años 90, comenzaron a aparecer varios reportes de caso que relacionaban las fluoroquinolonas con neuropatía periférica ${ }^{32,33}$, asociación que ha ido aumentando en evidencia en el último tiempo ${ }^{34}$. El año 2013 la Agencia Norteamericana de Alimentos y Medicamentos de E.U.A., emitió un comunicado indicando que todos los folletos de información de fluoroquinolonas debían colocar la neuropatía periférica como un efecto adverso grave, aunque infrecuente ${ }^{35}$. El inicio de síntomas tiene una latencia variable, pero más de la mitad de los pacientes inician síntomas dentro de la semana de uso $^{36,37}$. La sintomatología presentada es bien amplia, con síntomas sensoriales más que motores, destacando entre las alteraciones sensoriales: parestesias, entumecimiento, dolor urente, sensación de agujas o golpes eléctricos, hiperestesia, hipoestesia y alodinia; y entre las alteraciones motoras: debilidad, fasciculaciones, temblores, espasmos y contracciones ${ }^{37}$. En varios casos puede llegar a ser intensa y debilitante, e incluso, se han descrito cuadros irreversibles en algunos pacientes ${ }^{38}$. No se conocen factores de riesgo, ni claramente su mecanismo y frente a su aparición es necesario descartar la influencia de otros antimicrobianos que pueden producir este problema, como metronidazol, linezolid y nitrofurantoína ${ }^{39}$. Si el paciente desarrolla síntomas de neuropatía periférica, debe interrumpirse inmediatamente la administración de la fluoroquinolona.

\section{Interacciones con otros medicamentos y seguridad}

Las interacciones de las fluoroquinolonas con otros medicamentos pueden ser clasificadas como farmacocinéticas o farmacodinámicas. Sólo consideremos interacciones que afectan la seguridad de cada medicamento y no aquellas que reducen el efecto terapéutico.

Las interacciones farmacocinéticas involucran principalmente alteraciones de un medicamento por otro en términos de absorción, distribución, metabolismo y excreción. En el hígado ocurren las principales interacciones mediante la competencia de las fluoroquinolonas con los otros medicamentos por los citocromos $\mathrm{P} 450$, enzimas encargadas de la metabolización de xenobióticos ${ }^{40}$ (Tabla 2).

Las interacciones farmacodinámicas involucran alteraciones del efecto de un medicamento sobre otro. Al igual que otros antimicrobianos, las quinolonas pueden aumentar el efecto de los anticoagulantes orales warfarina y acenocumarol ${ }^{44}$. Se ha postulado desplazamiento del anticoagulante de la unión a proteínas plasmáticas, inhibición de citocromo y disrupción de la microbiota intestinal que sintetiza vitamina K; por lo que su mecanismo involucra interacción farmacocinética y farmacodinámica ${ }^{46}$. Se aconseja una monitorización frecuente del INR durante y justo después de la administración concomitante con una quinolona con un agente anticoagulante oral ${ }^{45}$. Otras interacciones farmacodinámicas involucran potenciación del

Tabla 2. Medicamentos que pueden aumentar sus concentraciones sanguíneas en presencia de ciprofloxacina

\begin{tabular}{ll}
\hline Grupo & Representantes del grupo \\
Antipsicóticos & Clozapina, olanzapina, haloperidol \\
Antidepresivos & Amitriptilina, imipramina, clomipramina, duloxetina \\
\hline Cardiovascular & Verapamilo, propranolol \\
Broncodilatadores & Teofilina \\
Relajantes musculares & Tizanidina \\
Anestésicos & Ropivacaina, lidocaina \\
\hline
\end{tabular}




\begin{tabular}{|c|c|c|}
\hline & Dosis en niños & Dosis adolescentes/adultos \\
\hline \multicolumn{3}{|l|}{ Ciprofloxacina } \\
\hline Oral & 20-40 mg/kg de peso/día, cada $12 \mathrm{~h}$ & 500 mg cada 12 h (máximo 750 mg cada 12 h) \\
\hline Intravenoso & 20-30 mg/kg de peso/día cada 8 o 12 h & 400 mg cada 12 h (máximo 400 mg cada 8 h) \\
\hline \multicolumn{3}{|l|}{ Levofloxacina } \\
\hline Oral/intravenosa & $\begin{array}{l}6 \text { meses- } 5 \text { años: } 16-20 \mathrm{mg} / \mathrm{kg} \text { de peso/día, cada } 12 \mathrm{~h} \\
\text { Mayor de } 5 \text { años: } 10 \mathrm{mg} / \mathrm{kg} \text { de peso/día, } 1 \text { vez al día }\end{array}$ & $500-750$ mg/día, cada 24 h \\
\hline \multicolumn{3}{|l|}{ Moxifloxacina } \\
\hline Oral/intravenosa & Datos limitados & 400 mg/día, cada 24 h \\
\hline
\end{tabular}

riesgo de prolongación del intervalo QT de la quinolona con medicamentos arritmogénicos ${ }^{47}$.

\section{Fluoroquinolonas en niños}

Desde hace muchos años, las quinolonas son evitadas en niños por sus supuestas propiedades nocivas sobre el crecimiento $^{48}$. Hoy se acepta que no deben ser utilizadas en pacientes pediátricos para infecciones de rutina cuando existe otro antimicrobiano seguro y sólo deberían ser consideradas en infecciones graves, sin otra alternativa de tratamiento ${ }^{49,50}$. Las recomendaciones de uso de fluoroquinolonas en niños por la Academia Americana de Pediatría (AAP) en el año 2016, son las siguientes ${ }^{51}$ :

- Exposición a Bacillus anthracis (también aprobado por FDA).

- Infecciones del tracto urinario causadas por Pseudomonas aeruginosa $\mathrm{u}$ otros bacilos gramnegativos multiresistentes. (FDA lo tiene licenciado para infecciones del tracto urinario complicadas y pielonefritis causadas por Escherichia coli).

- Otitis media supurativa crónica u otitis externa maligna por Pseudomonas aeruginosa.

- Osteomielitis aguda o crónica causada por P. aeruginosa.

- Exacerbación pulmonar en pacientes con fibrosis quística colonizados con $P$. aeruginosa.

- Infecciones por micobacterias susceptibles.

- Infecciones por bacilos gramnegativos en pacientes inmunosuprimidos en que hay resistencia a otros antimicrobianos alternativos.

- Infecciones del tracto gastrointestinal, causadas por especies multi-resistentes como Shigella spp, Salmonella spp, Vibrio cholerae o Campylobacter spp.

- Infecciones serias atribuibles a agentes susceptibles a quinolonas en niños con alergia severa a antimicrobianos habituales.

Las dosis han sido establecidas para este grupo etario (Tabla 3).
En general, el perfil de seguridad de las fluoroquinolonas es similar a los adultos. Un estudio prospectivo, multicéntrico, observacional, de cohorte, que compara efectos adversos potenciales en 276 pacientes pediátricos que recibieron fluoroquinolonas y 249 controles que recibieron otro antimicrobiano encontró un odds ratio para potenciales efectos adversos en el grupo de fluoroquinolonas de 3,7 (95\% IC, 1,9 a 7,5 $)^{52}$. El sistema afectado más frecuentemente fue el gastrointestinal, seguido de alteraciones músculo-esqueléticas (artralgias y mialgias pero no tendinopatía) OR: 9,3 (95\% IC, 1,2 a 195), piel y $\mathrm{SNC}^{52}$. Respecto a las alteraciones músculo-esqueléticas, ya comentadas previamente, en teoría el riesgo de alteraciones de cartílago sería más alto porque el paciente pediátrico está en crecimiento. En una revisión sistemática de seguridad de ciprofloxacina en 16.184 niños de 105 estudios, ocurrieron 258 efectos adversos músculo-esqueléticos en 232 pacientes (riesgo estimado 1,6:95\% IC, 0,9 a 2,6), con un efecto adverso músculo-esquelético por cada 62,5 pacientes $^{53}$. El 50\% correspondía a artralgias, siendo más frecuentemente afectada la rodilla. Estos eventos eran reversibles al suspender el antimicrobiano.

Por último, destacamos que, al igual que en adultos, existen otros potenciales efectos adversos pero que ocurren con poca frecuencia en niños, como son alteraciones del sistema nervioso central (cefalea, convulsiones, mareos, alteraciones del sueño), neuropatía periférica, alteraciones en la homeostasis de la glucosa (hipo o hiperglicemia), prolongación del intervalo QT y disfunción hepática $^{51}$.

\section{Comentarios finales}

Las reacciones adversas graves a fluoroquinolonas constituyen una importante complicación del tratamiento de pacientes, tanto adultos como niños. La presente revisión aborda las reacciones adversas graves que producen las fluoroquinolonas sobre cinco sistemas: 
músculo-esquelético, endocrino, cardiovascular, nervioso central y periférico. Si bien los eventos mencionados son de frecuencias consideradas bajas, existen riesgos que los tratantes prescriptores deben conocer, de forma de lograr una detección temprana y prevenir la progresión de estos eventos en los pacientes. En Chile contamos con un sistema de farmacovigilancia de la Agencia Nacional de Medicamentos que recopila estos eventos ${ }^{54}$ y que nos ha permitido conocer que los antimicrobianos son uno de los grupos causantes de RAM más importantes a lo largo del país ${ }^{55}$. Es de gran importancia promover el reporte de las reacciones adversas a nuestra autoridad regulatoria, ya que son nuestros datos locales los que podrían permitirnos establecer acciones regulatorias nacionales que permitan mejorar optimizar el uso de este grupo de antibióticos.

\section{Resumen}

Las quinolonas constituyen una familia de antimicrobianos de amplio uso y si bien son consideradas segura para los pacientes, el conocimiento del perfil de seguridad es necesario para que los profesionales estén alertas a lo que deben vigilar. Sobre el sistema músculo-esquelético, las quinolonas tienen el potencial de dañar cartílagos, provocando incluso muy excepcionalmente rotura de tendón. A nivel endocrino se ha observado hipoglicemia/hiperglicemia, por lo que en pacientes diabéticos se recomienda el control cuidadoso de la glicemia. Las reacciones adversas cardiovasculares son poco frecuentes, pero pueden ir desde alteraciones del ECG como prolongación del QT sin traducción clínica a graves arritmias que pueden ser de riesgo vital. En el sistema nervioso, destaca la aparición de alteraciones del sistema nervioso central y la neuropatía periférica. Durante la evaluación de la seguridad de las quinolonas es importante considerar las potenciales interacciones con otros medicamentos. En niños se prefiere no usar las fluoroquinolonas debido al potencial riesgo de daño a los cartílagos de crecimiento, efectos que no parecen ser tan dramáticos a la luz de la evidencia actual. A pesar del optimismo se debe evaluar la seguridad del tratamiento de estos antimicrobianos en todo paciente pediátrico.

\section{Referencias bibliográfícas}

1.- Bavestrello F L, Cabello M A. Community antibiotic consumption in Chile, 2000-2008. Rev Chilena Infectol 2011; 28 (2): 107-12.

2.- Patel K, Goldman J L. Safety concerns surrounding quinolone use in children. J Clin Pharmacol 2016; 56 (9): 1060-75.

3.- Owens RC. QT prolongation with antimicrobial agents: understanding the significance. Drugs 2004; 64 (10): 1091-124.

4.- Yip C, Lee A J. Gatifloxacin-induced hyperglycemia: A case report and summary of the current literature. Clin Ther 2006; 28 (11): 1857-66.

5.- Zhanel G G, Walkty A, Vercaigne L, Karlowsky J A, Embil J, Gin A S, et al. The new fluoroquinolones: A critical review. Can J Infect Dis 1999; 10 (3): 207-38.

6.- OMS. Vigilancia de la seguridad de los medicamentos: Guía para la instalación y puesta en funcionamiento de una Centro de Farmacovigilancia. Uppsala: the Uppsala Monitoring Centre. [Internet]. 2001 [cited 2016 Dec 13]. Available from: https://es.scribd. com/document/320649249/Vigilancia-de-LaSeguridad-de-Los-Medicamentos.

7.- Sendzik J, Lode H, Stahlmann R. Quinoloneinduced arthropathy: an update focusing on new mechanistic and clinical data. Int J Antimicrob Agents 2009; 33 (3): 194-200.

8.- Bacci C, Galli L, de Martino M, Chiappini E. Fluoroquinolones in children: update of the literature. J Chemother 2015; 27 (5): 257-65.
9.- Budny A M, Ley A N. Fluoroquinolonemediated Achilles rupture: a case report and review of the literature. J Foot Ankle Surg 2015; 54 (3): 494-6.

10.- van der Linden P D, van Puijenbroek E P, Feenstra J, Veld B A, Sturkenboom M C, Herings R M, et al. Tendon disorders attributed to fluoroquinolones: a study on 42 spontaneous reports in the period 1988 to 1998 . Arthritis Rheum 2001; 45 (3): 235-9.

11.- Melhus A. Fluoroquinolones and tendon disorders. Expert Opin Drug Saf 2005; 4 (2): 299-309.

12.- Arabyat R M, Raisch D W, McKoy J M, Bennett C L. Fluoroquinolone-associated tendon-rupture: a summary of reports in the Food and Drug Administration's adverse event reporting system. Expert Opin Drug Saf 2015; 14 (11): 1653-60.

13.- Stephenson A L, Wu W, Cortes D, Rochon P A. Tendon injury and fluoroquinolone use: a systematic review. Drug Saf 2013; 36 (9): 709 21.

14.- Mohr J F, McKinnon P S, Peymann P J, Kenton I, Septimus E, Okhuysen P C. A retrospective, comparative evaluation of dysglycemias in hospitalized patients receiving gatifloxacin, levofloxacin, ciprofloxacin, or ceftriaxone. Pharmacotherapy 2005; 25 (10): 1303-9.

15.- Aspinall S L, Good C B, Jiang R, McCarren M, Dong D, Cunningham F E. Severe dysglycemia with the fluoroquinolones: a class effect? Clin Infect Dis 2009; 49 (3): 402-8.

16.- El Ghandour S, Azar S T. Dysglycemia associated with quinolones. Prim Care Diabetes
2015; 9 (3): 168-71.

17.- Ambrose P G, Bhavnani S M, Cirincione B B, Piedmonte M, Grasela T H. Gatifloxacin and the elderly: pharmacokinetic-pharmacodynamic rationale for a potential age-related dose reduction. J Antimicrob Chemother 2003; 52 (3): 435-40.

18.- Pugi A, Longo L, Bartoloni A, Rossolini G M, Mugelli A, Vannacci A, et al. Cardiovascular and metabolic safety profiles of the fluoroquinolones. Expert Opin Drug Saf 2012; 11 (1): 53-69.

19.- Frothingham R. Glucose homeostasis abnormalities associated with use of gatifloxacin. Clin Infect Dis 2005; 41 (9): 126976.

20.- Ishiwata Y, Sanada Y, Yasuhara M. Effects of gatifloxacin on serum glucose concentration in normal and diabetic rats. Biol Pharm Bull 2006; 29 (3): 527-31.

21.- Elming H, Brendorp B, Køber L, Sahebzadah $\mathrm{N}$, Torp-Petersen C. QTc interval in the assessment of cardiac risk. Card Electrophysiol Rev 2002; 6 (3): 289-94.

22.- Frommeyer G, Eckardt L. Druginduced proarrhythmia: risk factors and electrophysiological mechanisms. Nat Rev Cardiol 2015; 13 (1): 36-47.

23.- Ball P. Quinolone-induced QT interval prolongation: a not-so-unexpected class effect. J Antimicrob Chemother 2000; 45 (5): 557-9.

24.- Samaha F F. QTC interval prolongation and polymorphic ventricular tachycardia in association with levofloxacin. Am J Med 1999; 107 (5): 528-9. 
25.- Badshah A, Janjua M, Younas F, Halabi A R, Cotant J F. Moxifloxacin-induced QT prolongation and torsades: an uncommon effect of a common drug. Am J Med Sci 2009; 338 (2): 164-6.

26.- Barnes B J, Hollands J M. Drug-induced arrhythmias. Crit Care Med 2010; 38 (6 Suppl): S188-97.

27.- Stahlmann R. Clinical toxicological aspects of fluoroquinolones. Toxicol Lett 2002; 127 (1-3) 269-77.

28.- Stahlmann R, Lode H. Safety considerations of fluoroquinolones in the elderly. drugs aging 2010; 27 (3): 193-209.

29.- Bellon A, Pérez-García G, Coverdale J H, Chacko R C. Seizures associated with levofloxacin: case presentation and literature review. Eur J Clin Pharmacol 2009; 65 (10): 959-62.

30.- Kushner J M, Peckman H J, Snyder C R. Seizures associated with fluoroquinolones. Ann Pharmacother 2001; 35 (10): 1194-8.

31.- Gervasoni C, Cattaneo D, Falvella F S, Vitiello P, Cheli S, Milazzo L, et al. Levofloxacininduced seizures in a patient without predisposing risk factors: the impact of pharmacogenetics. Eur J Clin Pharmacol 2013; 69 (8): 1611-3.

32.- Aoun M, Jacquy C, Debusscher L, Bron D, Lehert M, Noel P, et al. Peripheral neuropathy associated with fluoroquinolones. Lancet 1992 ; 340 (8811): 127.

33.- Rollof J, Vinge E. Neurologic adverse effects during concomitant treatment with ciprofloxacin, NSAIDS, and chloroquine: possible drug interaction. Ann Pharmacother 1993; 27 (9): 1058-9.

34.- Etminan M, Brophy J M, Samii A. Oral fluoroquinolone use and risk of peripheral neuropathy: a pharmacoepidemiologic study. Neurology 2014; 83 (14): 1261-3.

35.- FDA. FDA Drug Safety Communication: FDA requires label changes to warn of risk for possibly permanent nerve damage from antibacterial fluoroquinolone drugs taken by mouth or by injection [Internet]. 8/15/2013. [citado el 13 de diciembre de 2016]. Available from: http:/www.fda.gov/Drugs/DrugSafety/ ucm365050.htm

36.- Hedenmalm K, Spigset O. Peripheral sensory disturbances related to treatment with fluoroquinolones. J Antimicrob Chemother 1996; 37 (4): 831-7.

37.- Cohen J S. Peripheral neuropathy associated with fluoroquinolones. Ann Pharmacother 2001; 35 (12): 1540-7.

38.- Francis J K, Higgins E. Permanent peripheral neuropathy: a case report on a rare but serious debilitating side-effect of fluoroquinolone administration. J Investig Med High Impact Case Reports 2014; 2 (3): 2324709614545225.

39.- Vilholm O J, Christensen A A, Zedan A H, Itani M. Drug-induced peripheral neuropathy. Basic Clin Pharmacol Toxicol 2014; 115 (2): 185-92.

40.- Dresser G K, Spence J D, Bailey D G. Pharmacokinetic-pharmacodynamic consequences and clinical relevance of cytochrome P450 3A4 inhibition. Clin Pharmacokinet 2000; 38 (1): 41-57.

41.- Fish D N, Chow A T. The clinical pharmacokinetics of levofloxacin. Clin Pharmacokinet 1997; 32 (2): 101-19.

42.- Moise P A, Birmingham M C, Schentag J J. Pharmacokinetics and metabolism of moxifloxacin. Drugs Today (Barc) 2000; 36 (4): 229-44.

43.- Al-Omar M A. Ciprofloxacin: drug metabolism and pharmacokinetic profile. In: Profiles of drug substances, excipients, and related methodology 2005. p. 209-14.

44.- Douros A, Grabowski K, Stahlmann R. Safety issues and drug-drug interactions with commonly used quinolones. Expert Opin Drug Metab Toxicol 2014; 11 (1): 1-15.

45.- Bolhuis M S, Panday P N, Pranger A D, Kosterink J G W, Alffenaar J-WC. Pharmacokinetic drug interactions of antimicrobial drugs: a systematic review on oxazolidinones, rifamycines, macrolides, fluoroquinolones, and beta-lactams. Pharmaceutics 2011; 3 (4): 865-913.
46.- Schelleman H, Bilker W, Brensinger C, Han $\mathrm{X}$, Kimmel S, Hennessy S. Warfarin with fluoroquinolones, sulfonamides, or azole antifungals: interactions and the risk of hospitalization for gastrointestinal bleeding. Clin Pharmacol Ther 2008; 84 (5): 581-8.

47.- Tisdale J E. Drug-induced QT interval prolongation and torsades de pointes: Role of the pharmacist in risk assessment, prevention and management. Can Pharm J / Rev des Pharm du Canada 2016; 149 (3): 139-52.

48.- Forsythe C T, Ernst M E. Do fluoroquinolones commonly cause arthropathy in children? CJEM 2007; 9 (6): 459-62.

49.- Choi S-H, Kim E Y, Kim Y-J. Systemic use of fluoroquinolone in children. Korean J Pediatr 2013; 56 (5): 196-201.

50.- Sabharwal V, Marchant C D. Fluoroquinolone use in children. Pediatr Infect Dis J 2006; 25 (3): 257-8.

51.- Jackson M A, Schutze G E, Committee on Infectious Diseases. The use of systemic and topical fluoroquinolones. Pediatrics 2016; 138 (5): e20162706-e20162706.

52.- Chalumeau M, Tonnelier S, D’Athis P, Tréluyer J-M, Gendrel D, Bréart G, et al. Fluoroquinolone safety in pediatric patients: a prospective, multicenter, comparative cohort study in France. Pediatrics 2003; 111 (6 Pt 1): e714-9.

53.- Adefurin A, Sammons H, Jacqz-Aigrain E, Choonara I. Ciprofloxacin safety in paediatrics: a systematic review. Arch Dis Child 2011; 96 (9): 874-80.

54.- Instituto de Salud Pública de Chile. Subdepartamento de Farmacovigilancia | Instituto de Salud Pública de Chile [Internet]. [citado el 17 de agosto de 2017]. Available from: http://www.ispch.cl/anamed/ subdeptofarmacovigilancia.

55.- Vergara Galván V. ISP. Simposio «Realidades del Tratamiento Antimicrobiano». Efectos Adversos de los Antibióticos II: ¿Existe registro? ¿Debemos notificar? ¿Cuándo y Como? [Internet]. [citado el 17 de agosto de 2017]. 